\title{
Características morfológicas da casca do ovo de avestruz ${ }^{1}$
}

\author{
Juliana P. Gamba ${ }^{2}$, Neide S. C. Sabino ${ }^{2}$, Manoel Garcia Neto ${ }^{2}$ e Wilson M. de Souza ${ }^{2}$ \\ ABSTRACT.- Gamba J.P., Sabino N.S.C., Garcia Neto M. \& Souza W.M. 2012. [Morphologi- \\ cals characteristics of the ostrich eggshell.] Características morfológicas da casca do ovo \\ de avestruz. Pesquisa Veterinária Brasileira 32(1):96-98. Departamento de Apoio, Produ- \\ ção e Saúde Animal, Curso de Medicina Veterinária, Faculdade de Odontologia Araçatuba, \\ Universidade Estadual Paulista, Rua Clóvis Pestana 793, Araçatuba, SP 16050-680, Brazil. \\ E-mail: juliana_gamba@yahoo.com.br \\ Morphological analysis of the ostrich eggshell is important because several factors can be \\ related to morphological hatchability of eggs of this species in Brazil. Therefore, 60 samples of \\ apparently normal eggs were analyzed (eggs hatched and with embryonic death). The obser- \\ vations were allowed to establish that: the average thickness of the eggshell, the porosity of \\ the shell of the eggs, and correlate these variables with hatchability. The results were statisti- \\ cally analyzed by Duncan's test, which showed that eggs with less porosity also have a lower \\ hatchability. The average porosity of hatched eggs is 19.87 pores $/ \mathrm{cm}^{2}$, and of eggs with em- \\ bryonic death is pores $/ \mathrm{cm}^{2} 16.78$. There was no statistical difference between the thickness \\ of the shell in the regions studied, no interaction between viability and the regions studied.
}

INDEX TERMS: Ostrich, eggshell, rind morphology, reproduction.

RESUMO.- A análise morfológica da casca do ovo da avestruz é importante, pois diversos fatores morfológicos podem estar relacionados com a eclodibilidade do ovo desta espécie no Brasil. Para tanto foram analisadas 60 amostras de ovos aparentemente normais (ovos eclodidos e com morte embrionária). Foram efetuadas observações que permitiram estabelecer: a espessura média da casca do ovo, a porosidade da casca destes ovos, e correlacionar estas variáveis com a eclodibilidade. Os resultados foram analisados estatisticamente mediante o teste de Duncan, o que mostrou que ovos com menor porosidade tem também uma menor eclodibilidade. A porosidade média dos ovos eclodidos é de 19,87 poros $/ \mathrm{cm}^{2}$, e dos ovos com morte embrionária é de 16,78 poros $/ \mathrm{cm}^{2}$. Não houve diferença estatística entre a espessura da casca nas regiões estudada, nem interação entre viabilidade e as regiões.

TERMOS DE INDEXAÇÃO: Avestruz, ovo, morfologia da casca, reprodução.

\section{INTRODUÇÃo}

$\mathrm{Na}$ tentativa de otimizar a postura e produtividade das fêmeas reprodutoras, é realizada a incubação artificial dos

\footnotetext{
${ }^{1}$ Recebido em 9 de fevereiro de 2011

Aceito para publicação em 19 de outubro de 2011.

${ }^{2}$ Departamento de Apoio, Produção e Saúde Animal, Faculdade de Odontologia Araçatuba (FOA), Curso de Medicina Veterinária, Universidade Estadual Paulista (Unesp), Rua Clóvis Pestana 793, Araçatuba, SP 16050680, Brasil.*Autor para correspondência: juliana_gamba@yahoo.com.br
}

ovos, contudo, não se tem conseguido boas taxas de eclosão, quando comparadas às taxas de incubação natural. Em virtude de reduzida realização de pesquisas referentes à incubação de ovos de avestruzes nestes últimos anos, são escassos os relatos sobre quais seriam as melhores condições para se incubar tais ovos.

Sabe-se que características físicas e funcionais dos ovos de avestruzes e de sua casca, assim como em outras espécies aviárias, variam na dependência do genótipo, idade da fêmea, condições e tempo de estocagem, temperatura, umidade relativa, gases ambientais, posicionamento do ovo, freqüência de viragens, nutrientes dos ovos, período da estação reprodutiva, condição nutricional e sanitária dos reprodutores (Ar \& Rahn 1985). Essas características se correlacionam, interferindo no índice de perda de peso do ovo (provavelmente em grande parte do albúmen), morte embrionária e, portanto, na eclodibilidade (Nahm 2001, Sahan et al. 2003). Qualquer anormalidade na interação destas variáveis pode alterar a função biológica do ovo, por exemplo, as trocas gasosas são influenciadas pelo peso do ovo, índice da forma, espessura e porosidade da casca (Gonzales et al. 1999, Hassan et al. 2005), assim como a perda de massa durante a incubação pode ser correlacionada positivamente com a porosidade (Estrada et al. 2006) e negativamente com o período de incubação (Peebles \& McDaniel 2004).

Sabe-se também que a espessura da casca do ovo varia de acordo com o habitat da ave (Jarvis et al. 1985), e esta variá- 
vel juntamente com a porosidade da casca determinam a sobrevida do embrião, sendo que ovos com baixa porosidade e uma menor espessura da casca tem uma menor eclodibilidade (Satteneni \& Satterlee 1994, Narushin \& Romanov 2002).

Não diferente do que ocorre em outros países, no Brasil muito pouco se relata sobre esses fatores físicos e funcionais dos ovos de avestruzes e suas relações com as condições de incubação artificial praticadas.

Este trabalho foi realizado com o objetivo de avaliar a espessura e a porosidade da casca do ovo em diversas regiões e correlacionar essas variáveis com a eclodibilidade dos ovos.

\section{MATERIAL E MÉTODOS}

Foram analisadas 60 amostras de ovos de diferentes procedências, aparentemente normais, 30 ovos eclodidos e 30 com morte embrionária (de causa não determinada).

A porosidade dos ovos foi obtida através da análise de três fragmentos de $1 \mathrm{~cm}^{2}$ cada, retiradas da região da câmara de ar, da região do equador e do pólo inferior. Para melhor visualização e acurácia na contagem dos poros, as amostras foram tingidas usando corante azul de metileno e analisadas com auxílio de uma lupa. Foram contados apenas os grandes poros, conforme descrito por Gonzalez et al. (1999).

A média da espessura da casca, expressa em milímetros, foi obtida pela mensuração das mesmas amostras utilizada para avaliar a porosidade. As membranas internas da casca foram removidas antes da mensuração, que foi realizada com paquímetro.

0 delineamento experimental utilizado foi inteiramente casualizado, com fatorial 2x3: eclodibilidade (eclodidos e morte embrionária) e regiões da casca do ovo (Câmara de ar, Equatorial e Pólo Inferior). A análise de variância foi realizada segundo os procedimentos do PROC GLM do sistema SAS (2000), e as médias, comparadas pelo teste de Duncan a 5\% de probabilidade.

Para ilustração deste trabalho foram confeccionados esquemas e obtidas algumas imagens mediante equipamentos que permitiram ampliações de até $20 x$.

\section{RESULTADOS}

Os valores para o número de poros e espessura da casca dos ovos segundo a viabilidade (eclodido e não eclodido) e a região (câmara de ar, equatorial e pólo inferior) são apresentados no Quadro 1.

Quadro 1. Valores médios e análise de variância do número de poros e espessura da casca de ovos segundo a viabilidade

(Eclodidos ou Morte embrionária), e a região estudada (Câmara de ar, Equador e Pólo inferior)

\begin{tabular}{lcc}
\hline Variáveis & Poros & Espessura \\
\hline & \multicolumn{1}{c}{ Viabilidade } \\
Eclodidos & $19,87^{\mathrm{a}}$ & $1,78^{\mathrm{Ns}}$ \\
Morte embrionária & $16,78^{\mathrm{b}}$ & $1,81^{\mathrm{Ns}}$ \\
Câmara ar & $19,62^{\mathrm{Ns}}$ & $1,82^{\mathrm{Ns}}$ \\
Equador & $17,85^{\mathrm{Ns}}$ & $1,77^{\mathrm{Ns}}$ \\
Pólo inferior & $17,40^{\mathrm{Ns}}$ & $1,82^{\mathrm{Ns}}$ \\
& 0,0003 & \\
Viabilidade (V) & 0,1323 & 0,1595 \\
Região (R) & 0,9012 & 0,1240 \\
VxR & 34,19 & 0,9535 \\
C. V. (\%) & 9,48 \\
\hline
\end{tabular}

$\overline{\mathrm{a}, \mathrm{b}}$ Médias seguidas de letras diferentes na mesma coluna, dentro de cada variável, diferem entre si pelo teste de Duncan a $5 \%$ de probabilidade. C.V. = Coeficiente de Variação. ns Não significativo
A análise demonstrou a influência negativa de uma baixa porosidade dos ovos sobre a eclodibilidade. Os ovos não eclodidos são aqueles que possuem uma menor quantidade de poros. A porosidade média dos ovos eclodidos é de 19,87 poros $/ \mathrm{cm}^{2}$, e dos ovos com morte embrionária é de 16,78 poros $/ \mathrm{cm}^{2}$. Não houve interação entre a viabilidade e as regiões da casca do ovo para a porosidade. (Quadro 1).
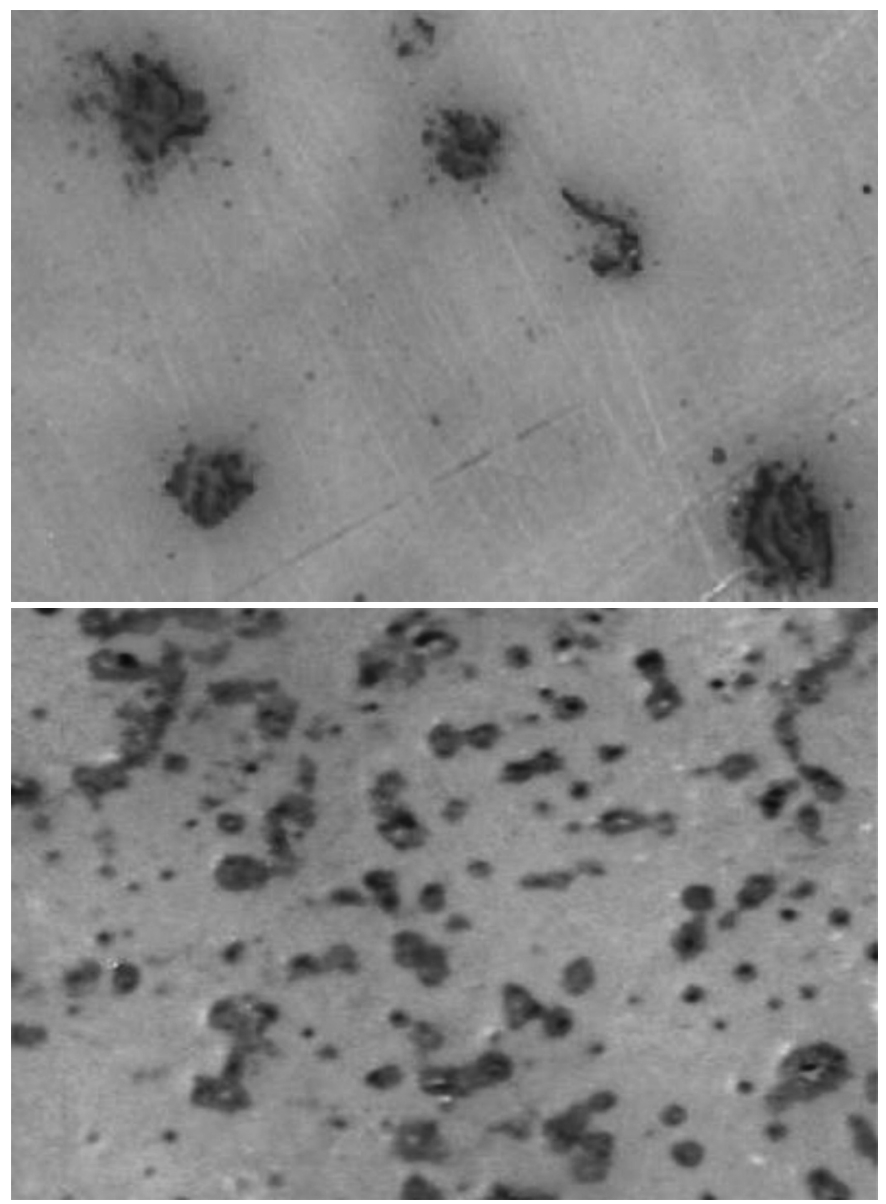

Fig.1. Cascas de ovos eclodidos e de Morte embrionária, respectivamente, na região da Câmara de ar demonstrando os diferentes tipos de poros, onde cada região azul escura representa um poro. $20 \mathrm{x}$.

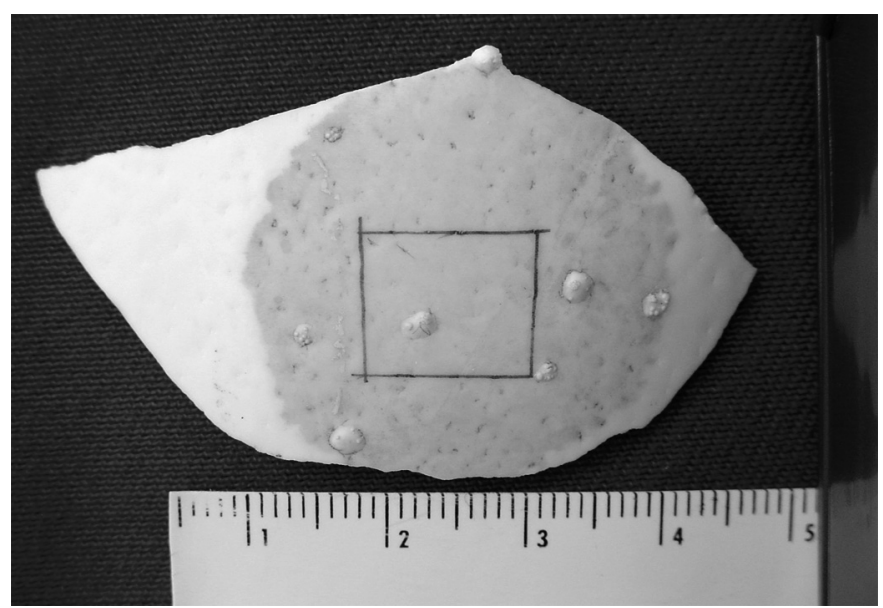

Fig.2. Casca de ovo de morte embrionária com irregularidades na superfície. 
A espessura média da casca dos ovos foi $1,80 \mathrm{~mm}$ em ovos de morte embrionária, e 1,78mm em ovos eclodidos, não houve interação entre viabilidade e as regiões da casca do ovo, não havendo diferença estatística entre as regiões estudadas, nem entre ovos eclodidos e de morte embrionária. Não houve interação entre viabilidade e as regiões da casca do ovo (Quadro 1).

$\mathrm{Na}$ avaliação das cascas dos ovos na lupa, foi possível verificar que ovos eclodidos possuem poros maiores e melhor delineados, principalmente na região da câmara de ar; ao contrário dos ovos de morte embrionária que possuem poros menores e irregulares (Fig.1).

Em algumas amostras de ovos não eclodidos foi possível observar irregularidades na casca do ovo (Fig.2), assim como em outras amostras foram encontrados inúmeros pequenos poros, não sendo possível determinar a porosidade de tais cascas.

\section{DISCUSSÃO}

De acordo com os resultados deste trabalho, o número de poros está relacionado na eclodibilidade de ovos de avestruzes (Satteneni \& Satterlee 1994, Gonzales et al. 1999). A eclodibilidade é menor em ovos com menor porosidade (Hicks-Alldrege 1998, Abbaspour-Fard et al. 2010), o que também foi observado por Satteneni \& Satterlee (1994), relatando que, a redução de área porosa é responsável pela menor difusão gasosa, essencial para a sobrevivência da ave durante a incubação (Deeming et al. 1993, Gonzales et al. 1999).

A relação da disposição dos poros e a eclodibilidade não foi encontrada nesse experimento, assim como Wagner-Amos \& Seymour (2002) relatou que o crescimento embrionário independe da distribuição dos poros na casa do ovo.

A espessura da casca do ovo do presente estudo $(1,79$ $1,83 \mathrm{~mm}$ ) foi similar à média já relatada em outros estudos (Madrid et al. 1996, Carrer et al. 2004). Foi constatado que a espessura não afetou significativamente a eclodibilidade, assim como também demonstrado por Sahan et al. (2003).

Os ovos que apresentaram morte embrionária foram encontrados inúmeros poros pequenos e rasos, que não foram contados pois há uma incerteza de que esses pequenos poros são verdadeiros, ou seja, que atravessam toda a espessura da casca (Gonzales et al. 1999; Nahn 2001). Se estes poros não atravessam do lado externo da casca ao interno, possivelmente a causa da morte embrionária justifique-se pela falta de trocas gasosas e a não perda de massa durante a incubação, visto que a não perca de peso do ovo durante a incubação é uma das limitações para o desenvolvimento embrionário e, consequentemente, baixo índice de eclodibilidade dos ovos (Abbaspour-Fard et al. 2010). Segundo Rahn et al. (1979) as trocas gasosas entre o ambiente externo e a região interna do ovo, são diretamente proporcional à área de poros viáveis para esta difusão.

Ainda no presente experimento foram encontradas irregularidades na casca de alguns ovos de morte embrionária, e segundo Solomon (1997), atribui-se essa alteração ao maior tempo de permanência no útero, o que resulta em deposições adicionais de cálcio, criando áreas de maior espessura com irregularidades no trajeto dos poros, sendo observado que cada fêmea mantém o mesmo padrão de cor e característica semelhante para poros, o que evidência um padrão genético (Kim \& Schalk 2004).

\section{CONCLUSÃO}

A porosidade é uma característica que influencia a eclodibilidade de ovos de avestruzes, sendo que poros maiores e profundos têm relação direta com a viabilidade do embrião. Portanto, a porosidade da casca do ovo deve ser usada como ferramenta para seleção de matrizes, visando uma menor mortalidade embrionária durante a incubação.

\section{REFERÊNCIAS}

Abbaspour-Fard M.H., Emadi B. \& Aghkhani M.H. 2010. Fertility Recognition of Ostrich Egg Using Physical Properties. J. Appl. Sci. 10:1405-1412.

Ar A. \& Rahn H. 1985. Pores in avian eggshells: gas conductance, gas exchange and embryonic growth rate. Resp. Physiol. 61:1-20.

Carrer C.C, Elmôr R.A., Kornfeld M.E. \& Carvalho M.C. 2004. A criação do avestruz: guia completo de A a Z. Ostrich do Brasil, São Paulo. 255p.

Deeming D.C., Ayres A. \& Ayres F.J 1993. Observations on the commercial production of ostrich (Struthio camelus) in the United Kingdom: Incubation. Vet. Rec. 132:602-607.

Estrada M.A.J., Lopez J.A.Q. \& Rebolledo O.F.P. 2006. Effect of the initial weight and weight loss during incubation on the hatchability rate of ostrich eggs (Struthio camelus). Vet. Mexico 37(4):487-492.

Gonzalez A., Satterlee D.G., Mharer F. \& Cadd G.G. 1999. Factors affecting ostrich egg hatchability. Poult. Sci. 78:1257-1262.

Hassan S.M., Siam A.A., Mady M.E. \& Cartwright A.L. 2005. Egg storage period and weight effects on hatchability of ostrich (Struthio camelus) eggs. Poult. Sci. 84:1908-1912.

Hicks-Alldredge K.D. 1998. Ratite reproduction Vet. Clin. North Am. 14(3):437-453.

Kim L.B \& Schalk W.P.C. 2004. Genetic parameters for egg, chick and live weight traits recorded in farmed ostriches (Struthio camelus). Sci. Dir. 91:9-22.

Jarvis M.J.F., Jarvis C. \& Keffen R.H. 1985. Breeding seasons and laying patterns of the southern African Ostrich Struthio camelus. Ibis 127:442449.

Madrid A.V., Cenzano J. \& Vicente J.M. 1996. Manual de indústria dos alimentos. Varela, São Paulo, p.489-495.

Nahn K.H. 2001. Effects of storage length and weight loss during incubation on hatchability of ostrich eggs (Struthio camelus). Poult. Sci. 88:1667-1670.

Narushin V.G. \& Romanov M.N. 2002. Egg physical characteristics and hatchability. World's Poult. Sci. J. 58(3):297-303.

Peebles E.D. \& McDaniel C.D. 2004. A practical manual for understanding the shell structure of broiler hatching eggs and measurements of their quality. Office of Agricultural Communications. Mississippi State University, Bull. 1139, Apr. 2004. Accessed Sept. 25, 2010 <http://www.msstate.edu/dept/poultry/b1139.pdf>

Rahn H., Ar A. \& Paganelli C.V. 1979. How bird eggs breathe. Scient. Am. 240:46-55.

Sahan Ü., Altan Ö., Ipek A. \& Yilmaz B. 2003. Effect of some egg characteristics on the mass loss and hatchability of ostrich (Struthio camelus) eggs. Brit. Poult. Sci. 44(3):380-385.

Satteneni G. \& Satterlee D.G. 1994. Factors affecting hatchability of eggs. Poult. Sci. 73(1):38. (Resumo)

Solomon S.E. 1997. Egg and eggshell quality. lowa State University Press, Ames. 149p.

Wagner-Amos K. \& Seymour R.S. 2002. Effect of regional changes to shell conductance on oxygen consumption and growth of chicken embryos. Resp. Physiol. 129:385-395. 